\title{
A Study on Mental Health Assessments of College Students Based on Triangular Fuzzy Function and Entropy Weight Method
}

\author{
Jing Zhang $\mathbb{B}$ \\ Wuxi Institute of Technology Mechanical, Wuxi, Jiangsu 214121, China \\ Correspondence should be addressed to Jing Zhang; zhangjing@wxit.edu.cn
}

Received 23 October 2020; Revised 7 December 2020; Accepted 12 December 2020; Published 8 January 2021

Academic Editor: Yi-Zhang Jiang

Copyright ( 2021 Jing Zhang. This is an open access article distributed under the Creative Commons Attribution License, which permits unrestricted use, distribution, and reproduction in any medium, provided the original work is properly cited.

\begin{abstract}
The fuzziness and uncertainty of mental health assessments for college students lead to considerable challenges for psychological counselors in universities. Therefore, this paper proposes a method based on triangular fuzzy and entropy weights to evaluate the mental health of college students. This method not only reduces the interference of human factors but also realizes the quantification of the results of mental health evaluations among college students. The triangular fuzzy function and entropy weight were used to comprehensively evaluate the mental health of college students. The feasibility and rationality of the proposed method were verified by experiments on individual students and actual data from different colleges.
\end{abstract}

\section{Reviews}

Mental health education is an important part of comprehensively promoting high-quality education among college students, an important link in cultivating high-quality talent, and an important way to strengthen ideological education in colleges and universities. With the deepening of reform and opening up, we have to face many great changes in international and domestic environment. College students are facing increasing social competition and pressure including study, life, emotion, employment, and other aspects. These factors result in a variety of mental health problems of college students. Therefore, attention to the physical and mental health of college students is the focus of many schools and even the whole society.

Traditional evaluation methods cannot fully and reasonably consider various factors. Therefore, this article establishes a reasonable index system through the use of questionnaires to assess college students' physical and mental health. A fuzzy mathematics model is established, and the fuzzy mathematics and entropy weight methods are used to analyze college students' physical and mental health; to ensure that the evaluation results are scientific and accurate; to determine college students' mental health status through qualitative and quantitative analysis; to effectively improve and manage students' psychological problems; and to provide technical support to students.

Based on the characteristics and types of psychological crises among higher education students, a three-level network of crisis intervention is proposed that combines early warning, middle intervention, and late follow-up at different stages of psychological crisis [1]. A four-level crisis intervention network is established with a crisis intervention early warning system, a crisis intervention mechanism, various forms of psychological crisis prevention education, and a psychological crisis intervention mode for higher education students in the college system [2]. Fuzzy mathematics is used to evaluate the mental health of students. Based on the analysis of the data collected by the mental health measurement scale, a comprehensive mathematical evaluation model is established. The results of this study reflect the actual mental health of students [3]. Under the guidance of system thought, this paper proposes a network of mental health education experts [4].

An interactive network-based psychological consultation platform has previously been used to measure the mental health status of college students [5]. The expert-based student mental health education platform uses information technology, artificial intelligence, and decision support system theory [6-10]. To improve the mental health of 
college students, online interactive education has been widely used as a method of intervention [11, 12]. By using anonymous self-report data collected via the Internet, the relationship between college students' perception and personal mental health has been analyzed to evaluate the participants' perceived stigma, personal stigma, and social expectations [13]. Factors affecting the mental health of college students, including discrimination in housing and employment, reduction in the use of mental health services, and adverse mental health outcomes, have been analyzed, especially among ethnic minorities. Studies have suggested that more aggressive action should be taken to support African Americans and other specific communities [14-17].

To provide counselors with a scientific test that has unified and quantitative indicators of college students' mental health, this paper uses basic knowledge of fuzzy mathematics and adopts the method of multilevel fuzzy comprehensive evaluation theory to evaluate the psychological health of college students and establishes a fuzzy comprehensive evaluation model [18]. First, the factors that affect mental health and the commonly used grade indicators of mental health are identified. Second, a fuzzy comprehensive evaluation model of college students' mental health is established by successively constructing an evaluation index system, a set of factor importance, a fuzzy comprehensive evaluation matrix, and a fuzzy comprehensive evaluation set. Rational modeling of college students' mental health quality can enable an accurate analysis of their mental state and provide a theoretical basis for treating their mental state. An optimal modeling method of college students' mental health quality based on the entropy weight triangular fuzzy method is proposed. The theory of fuzzy clustering is used to analyze the mental health indicators of college students and the mental health status of students at different colleges and universities. The feasibility of the method is illustrated by combining the current data with data from the literature. The results show that the proposed model provides a strong basis for improving students' mental health.

\section{Index System of the Fuzzy Evaluation Model}

2.1. Determination of Factors. The questionnaire on physical and mental health of college students contains 50 items, divided into 10 factors, which are, respectively, as follows [19]:

(i) F1: intellectual characteristics (including 5 items 1 , $2,3,4$, and 5)

(ii) F2: emotional health characteristics (including items 6, 7, and 8)

(iii) F3: features of sound will (including 6 items 9, 10, $11,12,13$, and 14)

(iv) F4: personality integrity characteristics (including 8 items $15,16,17,18,19,20,21$, and 22)

(v) F5: characteristics of self-evaluation (including items $23,24,25$, and 26) (vi) F6: characteristics of harmonious interpersonal relationship (including items 27, 28, and 29)

(vii) F7: social adaptability characteristics (including five items no. 30332, 33, and 34)

(viii) F8: psychological activity characteristics (including 35,36 , and 37 )

(ix) F9: self-orientation (including 38, 39, 40, 41, 42, and 43)

(x) F10: additional items (including 44, 45, 46, 47, 48, 49 , and 50 , seven items)

Taking each of the above factors as an evaluation factor, the factor set was established as $U=\{F 1, F 2, F 3, F 4, F 5, F 6$, $F 7, F 8, F 9, F 10\}$.

2.2. Determination of the Evaluation Set. The physical and mental health of students is uncertain and fuzzy, so it is difficult to make accurate evaluation. We continuously have fixed interval variables which are used to indicate, namely, usually rating method, the assessment of students' physical and mental health status of the amount of spacing between calibration, such as five cases which were divided into I (good), II (better), III (general), IV (poor), and V (lower poor) five levels and establish evaluation set $\mathrm{V}=\{\mathrm{I}, \mathrm{II}, \mathrm{III}, \mathrm{IV}, \mathrm{V}\}$, corresponding to each and every project a symptom. There are five options including "no" (1 point), "light" ( 2 points), "medium" (3 points), "overweight" (4 points), and "serious" (5 points), corresponding to the above five levels.

\section{Fuzzy Weight}

3.1. Determination of Single-Factor Evaluation Matrix. The subjects were asked to choose among alternative answers for each item in the questionnaire. For each factor $F_{i}$, the number of times the respondents chose each answer was denoted as $a_{1}, a_{2}, a_{3}, a_{4}$, and $a_{5}$, and the normalized evaluation vector consisted of a single factor $a_{i 1}, a_{i 2}, a_{i 3}, a_{i 4}$, and $a_{i 5}, \quad$ in which $a_{i j}=\left(a_{j} / N_{i}\right)$, $(i=1,2, \ldots, 10, j=1,2,3,4,5)$ with $N_{i}$ being the total number of items contained in the factor. The ten singlefactor evaluation vectors were taken as row vectors to form the single-factor evaluation matrix [20]:

$$
R=\left[\begin{array}{ccc}
a_{11} & \cdots & a_{15} \\
\vdots & \ddots & \vdots \\
a_{10,1} & \cdots & a_{10,5}
\end{array}\right] .
$$

For each factor $F_{i}(i=1,2, \ldots, 10)$, we defined the factor score $b_{i}$ as follows:

$$
b_{i}=\frac{F_{i}}{\sum_{i=1}^{10} F_{i}} .
$$

The factor score obviously reflects the degree of the influence of the factor on the mental health of the tested person. Therefore, the weight vector is taken as follows: 


$$
A=\left(\frac{b_{1}}{\sum_{i=1}^{10} b_{i}}, \frac{b_{2}}{\sum_{i=1}^{10} b_{i}}, \ldots, \frac{b_{10}}{\sum_{i=1}^{10} b_{i}}\right) .
$$

The weight vector $A$ and the single-factor evaluation matrix $R$ are combined to evaluate mental health status. In the synthesis operation, in order to take into account each factor, the operator $M\left({ }^{\circ},+\right)$ is used to evaluate the vector $S=A^{\circ} R=\left(s_{1}, s_{2}, s_{3}, s_{4}, s_{5}\right)$. By comparing the five numbers of the vector $S$, if the number one $j$ is the largest, the mental health status of the subject is judged to be level $j$ according to the principle of maximum membership.

3.2. Analysis of Examples. Two hundred questionnaires were distributed to college students, and a total of 192 were recovered. The corresponding mathematical expectations were calculated on the basis of these 192 samples, and the weighted average method was used to obtain the weight vector for the mental health of college students. The statistics of the questionnaire subjects are shown in Table 1.

The weight vector is obtained: $A=(0.0981,0.0817$, $0.0899,0.1042,0.1226,0.1308,0.0785,0.0817,0.1144,0.0981$ ).

The single-factor evaluation matrix is as follows:

$$
R=\left[\begin{array}{ccccc}
0.400 & 0.200 & 0.400 & 0.000 & 0 \\
0.333 & 0.667 & 0.000 & 0.000 & 0 \\
0.333 & 0.333 & 0.167 & 0.167 & 0 \\
0.500 & 0.125 & 0.250 & 0.000 & 0.125 \\
0.250 & 0.250 & 0.250 & 0.250 & 0 \\
0.333 & 0.000 & 0.333 & 0.333 & 0 \\
0.400 & 0.400 & 0.000 & 0.200 & 0 \\
0.333 & 0.667 & 0.000 & 0.000 & 0 \\
0.500 & 0.000 & 0.167 & 0.333 & 0 \\
0.571 & 0.143 & 0.143 & 0.000 & 0.143
\end{array}\right] .
$$

The operator $M\left({ }^{\circ},+\right)$ is used to synthesize the heavy vector $A$ and the single factor $R$ evaluation matrix to obtain the evaluation vector $S=(0.3945,0.0 .2476,0.1876,0.1430$, 0.0271). The maximum membership degree principle is the students' physical and mental health level I (good). As the table shows, the most influential factors in the mental health status of college students are the characteristics of selfevaluation, followed by the characteristics of interpersonal harmony, self-orientation, intelligence, etc. The least influential factors are the characteristics of social adaptability.

3.3. Fuzzy Entropy Weight Evaluation Model. To measure differences in the mental health of college students, we selected the triangular fuzzy and entropy weight synthesis method. The mental health status of college students at different universities was ranked. The comprehensive scores were used to rank the mental health status of students at different universities.

Entropy is a measure of system state uncertainty and is used mainly to measure the amount of information contained in indicator data in an evaluation indicator system. The development of the nonfuzzy matrix $A$ is described as follows:

$$
A=\left[\begin{array}{ccc}
a_{11} & \cdots & a_{1 n} \\
\vdots & \ddots & \vdots \\
a_{n 1} & \cdots & a_{n n}
\end{array}\right] .
$$

If let $s_{i}=\sum_{i=1}^{10} a_{i j}(j=1,2,3,4,5)$ be the sum of the elements in the $i$ row, $P_{i j}=\left(a_{i j} / s_{i}\right)$ is defined as the probability of the element $a_{i j}$ in the matrix appearing in the row. Then, entropy of the probability matrix (2) can be obtained:

$$
\begin{gathered}
P=\left[\begin{array}{ccc}
P_{11} & \cdots & P_{1 n} \\
\vdots & \ddots & \vdots \\
P_{n 1} & \cdots & P_{n n}
\end{array}\right], \\
H_{i}=-\sum_{j=1}^{n} P_{i j} \log _{2}\left(P_{i j}\right), \quad(i=1,2, \ldots, n) .
\end{gathered}
$$

3.4. Definition and Operation Rules of Triangular Fuzzy Numbers. If $\mathrm{F}$ is a set of real numbers, F represents triangular fuzzy numbers, and $F \longrightarrow[0,1]$, it can be simply denoted as $M=(1, m, u)$, and its membership function $V(x)$ can be expressed as

$$
V(x)= \begin{cases}0, & x<a, \\ \frac{x-a}{m-a}, & a \leq x \leq m, \\ \frac{b-x}{b-m}, & m \leq x \leq b, \\ 0, & x>b .\end{cases}
$$

For the membership function $V(x), l<m<u$, the values of a and $b$ are the lower bound and the upper bound of the set $F$, respectively. $M_{1}=\left(l_{1}, m_{1}, u_{1}\right)$ and $M_{2}=\left(l_{2}, m_{2}, u_{2}\right)$ are defined as two fuzzy numbers of membership function $V(x)$. They satisfy the conditions $M_{1}+M_{2}=\left(l_{1}+l_{2}, m_{1}+m_{2}, u_{1}+\right.$ $\left.u_{2}\right)$ and $M_{1} \otimes M_{2}=\left(l_{1} \otimes l_{2}, m_{1} \otimes m_{2}, u_{1} \otimes u_{2}\right)$. For any real number $\lambda$, this formula $\lambda M=\lambda(l, m, u)=(\lambda l, \lambda m, u \lambda)$ is established.

Assume the intercept set $\beta \in[0,1]$ and use fuzzy numbers $\widetilde{1}, \widetilde{3}, \widetilde{5}$. The characteristic parameters and confidence interval are listed in Table 2.

By comparing the performance index score, use fuzzy number $\widetilde{1}, \widetilde{3}, \widetilde{5}$ to represent the relative strength of the elements of the judgment matrix. By determining the fuzzy weighted vector $\widetilde{w}$ and fuzzy judgment matrix $\widetilde{X}$, we get the general fuzzy judgment matrix $\widetilde{A}$, using fuzzy multiplied by the fuzzy judgment matrix $\widetilde{X}$ of each criterion layer: 
TABle 1: Statistical table of test subjects.

\begin{tabular}{|c|c|c|c|c|c|c|c|c|c|}
\hline \multirow{2}{*}{ Factor } & \multirow{2}{*}{ Total number of items } & \multicolumn{5}{|c|}{ The number of choices for the alternative answer } & \multirow{2}{*}{ Total score } & \multirow{2}{*}{ Factor score } & \multirow{2}{*}{ Weight } \\
\hline & & 1 point & 2 points & 3 points & 4 points & 5 points & & & \\
\hline 1 & 5 & 2 & 1 & 2 & 0 & 0 & 10 & 2.00 & 0.0981 \\
\hline 2 & 3 & 1 & 2 & 0 & 0 & 0 & 5 & 1.67 & 0.0817 \\
\hline 3 & 6 & 2 & 2 & 1 & 1 & 0 & 11 & 1.83 & 0.0899 \\
\hline 4 & 8 & 4 & 1 & 2 & 0 & 1 & 17 & 2.13 & 0.1042 \\
\hline 5 & 4 & 1 & 1 & 1 & 1 & 0 & 10 & 2.50 & 0.1226 \\
\hline 6 & 3 & 1 & 0 & 1 & 1 & 0 & 8 & 2.67 & 0.1308 \\
\hline 7 & 5 & 2 & 2 & 0 & 1 & 0 & 8 & 1.60 & 0.0785 \\
\hline 8 & 3 & 1 & 2 & 0 & 0 & 0 & 5 & 1.67 & 0.0817 \\
\hline 9 & 6 & 3 & 0 & 1 & 2 & 0 & 14 & 2.33 & 0.1144 \\
\hline 10 & 7 & 4 & 1 & 1 & 0 & 1 & 14 & 2.00 & 0.0981 \\
\hline Total & 50 & & & & & & & 20.39 & 1.0000 \\
\hline
\end{tabular}

TABLE 2: Characteristic parameters and confidence intervals of fuzzy numbers.

\begin{tabular}{lrr}
\hline Fuzzy numbers & Characteristic parameters & Confidence interval \\
\hline$\widetilde{1}$ & $(1,1,3)$ & {$[1,3-2 \beta]$} \\
$\widetilde{3}$ & $(1,3,5)$ & {$[1+2 \beta, 5-2 \beta]$} \\
$\widetilde{5}$ & $(3,5,7)$ & {$[3+2 \beta, 7-2 \beta]$} \\
\hline
\end{tabular}

$$
\widetilde{A}=\left[\begin{array}{cccc}
w_{11} a_{11} & w_{12} a_{12} & \cdots & w_{1 n} a_{1 n} \\
w_{21} a_{21} & w_{22} a_{22} & \cdots & w_{2 n} a_{2 n} \\
\vdots & \vdots & \ddots & \vdots \\
w_{n 1} a_{n 1} & w_{n 2} a_{n 2} & \cdots & w_{n n} a_{n n}
\end{array}\right]
$$

The total fuzzy judgment matrix is represented as $\widetilde{A}$. According to a given horizontal intercept set $\beta, \widetilde{A}$ will be expressed in an interval form:

$$
\widetilde{A}_{\beta}=\left[\begin{array}{ccc}
{\left[a_{11 l}^{\beta}, a_{11 r}^{\beta}\right]} & \cdots & {\left[a_{1 n l}^{\beta}, a_{1 n r}^{\beta}\right]} \\
{\left[a_{21 l}^{\beta}, a_{21 r}^{\beta}\right]} & \cdots & {\left[a_{2 n l}^{\beta}, a_{2 n r}^{\beta}\right]} \\
\vdots & \ddots & \vdots \\
{\left[a_{n 1 l}^{\beta}, a_{n 1 r}^{\beta}\right]} & \cdots & {\left[a_{n n l}^{\beta}, a_{n n r}^{\beta}\right]}
\end{array}\right] .
$$

If $\beta$ is given, the optimism indicator $\lambda$ is used to judge the matrix's satisfaction. $\lambda$ represents optimism level of policy makers. The larger value of $\lambda$ is the better. The matrix $\widetilde{A}_{\beta}$ can be converted the nonfuzzy matrix $\widetilde{\widetilde{A}}$ using $\lambda$.

$$
\tilde{A}=\left[\begin{array}{cccc}
\tilde{a}_{11} & \tilde{a}_{12} & \cdots & \tilde{a}_{1 n} \\
\tilde{a}_{21} & \tilde{a}_{22} & \cdots & \tilde{a}_{2 n} \\
\vdots & \vdots & \ddots & \vdots \\
\tilde{a}_{n 1} & \tilde{a}_{n 2} & \cdots & \tilde{a}_{n n}
\end{array}\right]
$$

According to equation (6), we get and normalize $H_{i}, i=1,2, \ldots, n$. Then, the entropy weight of the $i$ th factor is obtained.

$$
w_{H}^{i}=\frac{1-H_{i}}{\sum_{i=1}^{n}\left(1-H_{i}\right)} .
$$

Entropy weight $w_{H}^{i}(i=1,2, \ldots, n)$ can reflect the degree of mental health of college students under different factors.

\section{Comparison of Mental Health among Students from Different Universities}

4.1. Data Sources and Data Preprocessing. A total of 878 questionnaires were collected, with 100 questionnaires being distributed to each of 9 colleges. Then, 20 questionnaires were randomly selected from each university, and the scores of each factor were added. We analyzed the mental health status of college students at different universities by using the fuzzy entropy weight method. Table 3 shows the statistical data of the questionnaires from the different universities.

The fuzzy number interval of factor scores is calculated from equation (8) as shown in Table 4.

Factor scores of different schools universities can be converted to fuzzy number as shown in Table 5. 
TABLE 3: Factor scores of different schools universities.

\begin{tabular}{|c|c|c|c|c|c|c|c|c|c|c|}
\hline \multirow{2}{*}{ Factor } & \multirow{2}{*}{ Total number of items } & \multicolumn{9}{|c|}{ Factor scores of 9 universities } \\
\hline & & $\mathrm{U} 1$ & $\mathrm{U} 2$ & $\mathrm{U} 3$ & $\mathrm{U} 4$ & U5 & U6 & U7 & U8 & U9 \\
\hline 1 & 5 & 68.74 & 65.18 & 32.60 & 72.46 & 36.48 & 55.10 & 42.08 & 40.10 & 67.26 \\
\hline 2 & 3 & 42.48 & 72.46 & 57.64 & 22.86 & 22.54 & 50.52 & 54.38 & 40.40 & 40.34 \\
\hline 3 & 6 & 62.90 & 30.16 & 76.58 & 67.94 & 42.16 & 61.24 & 32.40 & 80.48 & 45.14 \\
\hline 4 & 8 & 24.82 & 53.08 & 58.84 & 74.72 & 23.70 & 23.62 & 33.00 & 75.92 & 39.48 \\
\hline 5 & 4 & 70.44 & 70.58 & 64.02 & 74.30 & 27.78 & 34.94 & 29.52 & 51.28 & 74.34 \\
\hline 6 & 3 & 43.02 & 27.80 & 31.36 & 60.62 & 25.88 & 59.18 & 41.86 & 56.74 & 48.42 \\
\hline 7 & 5 & 57.38 & 42.28 & 53.74 & 79.46 & 75.58 & 55.64 & 42.78 & 63.78 & 35.72 \\
\hline 8 & 3 & 23.70 & 63.76 & 53.26 & 51.38 & 45.36 & 31.70 & 47.24 & 31.08 & 40.08 \\
\hline 9 & 6 & 47.18 & 56.60 & 23.38 & 60.44 & 36.02 & 36.74 & 22.82 & 31.94 & 69.28 \\
\hline 10 & 7 & 28.82 & 77.20 & 76.76 & 33.70 & 22.76 & 40.38 & 21.90 & 40.60 & 57.86 \\
\hline Average score & 52.16 & 62.12 & 58.69 & 66.43 & 39.81 & 49.90 & 40.89 & 54.70 & 57.55 & \\
\hline
\end{tabular}

TABle 4: Fuzzy number.

\begin{tabular}{|c|c|c|c|c|c|c|c|}
\hline Factor & Min-value & Fuzzy value & Median & Fuzzy value & Median & Fuzzy value & Max-value \\
\hline 1 & 32.60 & $\tilde{1}$ & 45.89 & $\widetilde{3}$ & 59.17 & $\widetilde{5}$ & 72.46 \\
\hline 2 & 22.54 & $\tilde{1}$ & 39.18 & $\tilde{3}$ & 55.82 & $\widetilde{5}$ & 72.46 \\
\hline 3 & 30.16 & $\tilde{1}$ & 45.63 & $\tilde{3}$ & 61.11 & $\widetilde{5}$ & 80.48 \\
\hline 4 & 23.62 & $\tilde{1}$ & 41.05 & $\widetilde{3}$ & 58.49 & $\widetilde{5}$ & 75.92 \\
\hline 5 & 27.78 & $\tilde{1}$ & 43.30 & $\tilde{3}$ & 58.82 & $\widetilde{5}$ & 74.34 \\
\hline 6 & 25.88 & $\tilde{1}$ & 37.46 & $\widetilde{3}$ & 49.04 & $\widetilde{5}$ & 60.62 \\
\hline 7 & 35.72 & $\tilde{1}$ & 50.30 & $\tilde{3}$ & 64.88 & $\widetilde{5}$ & 79.46 \\
\hline 8 & 23.70 & $\tilde{1}$ & 37.05 & $\tilde{3}$ & 50.41 & $\widetilde{5}$ & 63.76 \\
\hline 9 & 22.82 & $\tilde{1}$ & 38.31 & $\tilde{3}$ & 53.79 & $\widetilde{5}$ & 69.28 \\
\hline 10 & 21.90 & $\tilde{1}$ & 40.33 & $\widetilde{3}$ & 58.77 & $\widetilde{5}$ & 77.20 \\
\hline Average & 39.81 & $\tilde{1}$ & 48.68 & $\tilde{3}$ & 57.56 & $\widetilde{5}$ & 66.43 \\
\hline
\end{tabular}

TABle 5: Fuzzy number.

\begin{tabular}{|c|c|c|c|c|c|c|c|c|c|}
\hline Factor & $U 1$ & $U 2$ & U3 & $U 4$ & U5 & U6 & U7 & U8 & U9 \\
\hline 1 & $\widetilde{5}$ & $\widetilde{5}$ & $\widetilde{1}$ & $\widetilde{5}$ & $\widetilde{1}$ & $\widetilde{5}$ & $\widetilde{1}$ & $\widetilde{1}$ & $\widetilde{5}$ \\
\hline 2 & $\tilde{3}$ & $\widetilde{5}$ & $\widetilde{5}$ & $\tilde{1}$ & $\tilde{1}$ & $\tilde{3}$ & $\tilde{3}$ & $\tilde{3}$ & $\tilde{3}$ \\
\hline 3 & $\widetilde{5}$ & $\widetilde{1}$ & $\widetilde{5}$ & $\widetilde{5}$ & $\widetilde{1}$ & $\widetilde{5}$ & $\widetilde{1}$ & $\widetilde{5}$ & $\widetilde{1}$ \\
\hline 4 & $\widetilde{1}$ & $\tilde{3}$ & $\widetilde{5}$ & $\widetilde{5}$ & $\widetilde{1}$ & $\widetilde{1}$ & $\widetilde{1}$ & $\widetilde{5}$ & $\widetilde{1}$ \\
\hline 5 & $\widetilde{5}$ & $\widetilde{5}$ & $\widetilde{5}$ & $\widetilde{5}$ & $\tilde{1}$ & $\tilde{1}$ & $\tilde{1}$ & $\widetilde{5}$ & $\widetilde{5}$ \\
\hline 6 & $\widetilde{3}$ & $\widetilde{1}$ & $\widetilde{1}$ & $\widetilde{5}$ & $\tilde{1}$ & $\widetilde{5}$ & $\tilde{3}$ & $\widetilde{5}$ & $\tilde{3}$ \\
\hline 7 & $\tilde{3}$ & $\tilde{1}$ & $\tilde{3}$ & $\widetilde{5}$ & $\widetilde{5}$ & $\tilde{3}$ & $\widetilde{1}$ & $\widetilde{3}$ & $\widetilde{1}$ \\
\hline 8 & $\tilde{1}$ & $\widetilde{5}$ & $\widetilde{5}$ & $\widetilde{5}$ & $\tilde{3}$ & $\tilde{1}$ & $\tilde{3}$ & $\tilde{1}$ & $\tilde{3}$ \\
\hline 9 & $\tilde{3}$ & $\widetilde{5}$ & $\widetilde{1}$ & $\widetilde{5}$ & $\widetilde{1}$ & $\widetilde{1}$ & $\widetilde{1}$ & $\widetilde{1}$ & $\widetilde{5}$ \\
\hline 10 & $\widetilde{1}$ & $\widetilde{5}$ & $\widetilde{5}$ & $\widetilde{1}$ & $\widetilde{1}$ & $\tilde{3}$ & $\widetilde{1}$ & $\tilde{3}$ & $\widetilde{5}$ \\
\hline Weight & $\tilde{3}$ & $\widetilde{5}$ & $\widetilde{5}$ & $\widetilde{5}$ & $\tilde{1}$ & $\tilde{3}$ & $\tilde{1}$ & $\tilde{3}$ & $\tilde{3}$ \\
\hline
\end{tabular}

4.2. Construction of the Fuzzy Weight Vector and Calculation of the Total Judgment Matrix. In different university management modes, the campus culture, management mechanism, and professional characteristics associated with university mental health influence are not identical, nor are the interactions of these factors. Therefore, to reflect different school factors and the influence of these factors on college students' mental health, Table 5 is averaged as a factor between the weight vector $\widetilde{w}=\left(\begin{array}{cccccccccc}U_{1} & U_{2} & U_{3} & U_{4} & U_{5} & U_{6} & U_{7} & U_{8} & U_{9} \\ & \widetilde{3} & \widetilde{5} & \widetilde{5} & \widetilde{5} & \widetilde{1} & \widetilde{3} & \widetilde{1} & \widetilde{3} & \widetilde{3}\end{array}\right)$, and based 
on formula (6) and Table 4, the fuzzy judgment matrix is transformed into a general judgment matrix. The total judgment matrix is as follows:

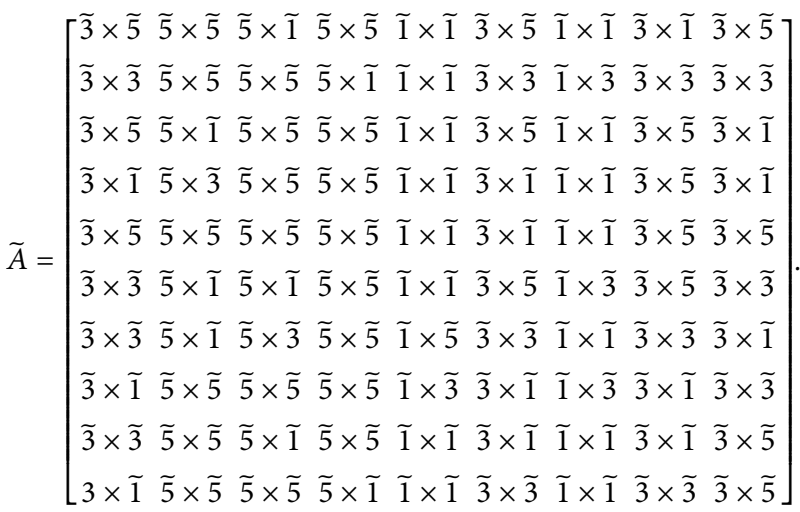

If $\beta=0.5$, by using formula (7), $\tilde{A}$ can be converted into an interval form.

$$
\widetilde{A}_{\beta}=\left[\begin{array}{ccccccccc}
{[8,24]} & {[16,26]} & {[4,6]} & {[16,36]} & {[1,1]} & {[8,24]} & {[1,1]} & {[2,4]} & {[8,24]} \\
{[4,16]} & {[16,36]} & {[16,36]} & {[4,18]} & {[1,1]} & {[4,16]} & {[2,4]} & {[4,16]} & {[4,16]} \\
{[8,24]} & {[4,6]} & {[16,36]} & {[16,36]} & {[1,1]} & {[8,24]} & {[1,1]} & {[8,24]} & {[2,4]} \\
{[2,4]} & {[8,24]} & {[16,36]} & {[16,36]} & {[1,1]} & {[2,4]} & {[1,1]} & {[8,24]} & {[2,4]} \\
{[8,24]} & {[16,36]} & {[16,36]} & {[16,36]} & {[1,1]} & {[2,4]} & {[1,1]} & {[8,24]} & {[8,24]} \\
{[4,16]} & {[4,6]} & {[4,6]} & {[16,36]} & {[1,1]} & {[8,24]} & {[2,4]} & {[8,24]} & {[4,16]} \\
{[4,16]} & {[4,6]} & {[16,36]} & {[16,36]} & {[4,6]} & {[4,16]} & {[1,1]} & {[4,16]} & {[2,4]} \\
{[2,4]} & {[16,36]} & {[16,36]} & {[16,36]} & {[2,4]} & {[2,4]} & {[2,4]} & {[2,4]} & {[4,16]} \\
{[4,16]} & {[16,36]} & {[4,6]} & {[16,36]} & {[1,1]} & {[2,4]} & {[1,1]} & {[2,4]} & {[8,24]} \\
{[2,4]} & {[16,36]} & {[16,36]} & {[4,6]} & {[1,1]} & {[4,16]} & {[1,1]} & {[4,16]} & {[8,24]}
\end{array}\right] .
$$

We let $\lambda=0.6$ and obtain the nonfuzzy judgment matrix. Formula (10) is used to transform the nonfuzzy judgment matrix into the probability matrix.

Then, formula (11) is used to obtain the synthesis score corresponding to the mental health status of every university student, as shown in Table 6 .

Table 6 shows that there are significant differences in college students' mental health and different factors reflect different aspects of college students' mental health.

4.2.1. Attaching Importance to the Psychological Health Assessment of College Students. College students' mental health assessments guide and create a foundation for college students' mental health education. Therefore, colleges and universities should prioritize college students' mental health assessments, improve mental health assessments, and establish more empirically based assessments. The analytical method based on fuzzy comprehensive evaluation can be used for reference. In addition, before the assessment of college students' mental health, college students should receive ideological education to enhance the importance of mental health assessment rather than simply completing the task and coping with it. Teachers should track and screen the evaluation results in a timely manner to achieve early detection, early intervention, and effective control.

4.2.2. Combining Mental Health Education with Ideological Education. Mental health education is the basis of ideological education, and ideological education can provide guidance for mental health education. Colleges and universities should pay attention to students' ideological dynamics and psychological characteristics and follow the laws of ideological education and mental health education so that ideological and psychological education promotes and complements each other [21]. In daily mental health education for college students, college teachers should treat the psychological and ideological problems of college students; integrate, guide, and educate the students and provide them with timely help.

4.2.3. Establishing Joint Mechanisms among Families, Schools, and Communities. For the mental health education of college students, relying only on school is not enough; the 
TABLE 6: Synthesis score of mental health status.

\begin{tabular}{lccccccccc}
\hline University & $U 1$ & $U 2$ & $U 3$ & $U 4$ & $U 5$ & $U 6$ & $U 7$ & $U 8$ & $U 9$ \\
\hline Synthesis score & 2.405 & 3.092 & 3.184 & 3.222 & 1.955 & 2.733 & 1.082 & 2.691 & 2.392 \\
\hline
\end{tabular}

role of parents is also extremely important. Colleges and universities should establish a joint mechanism with parents to understand the psychological dynamics of students at home and provide information about their psychological dynamics at school to parents to analyze the psychological health of students at both home and school in a more targeted approach $[22,23]$. Such a home-school joint mechanism should be normalized. Colleges and parents should communicate with each other, pay attention to the mental health of college students, and train them together.

\section{Conclusion}

This paper studies the application of fuzzy comprehensive evaluation to the mental health assessment of college students and establishes a corresponding fuzzy comprehensive evaluation model of the mental health of college students. This model of mental health evaluation, which is relatively new in mental health research, can greatly reduce the difficulty of psychological counseling workers in evaluating mental health and is more conducive to the development of psychological counseling.

Although the tools and methods used to quantify college students' mental health have developed greatly, the theory and technology of the quantification of risk in specific applications are not practicable, and the applicability of the methods is also greatly limited. In this paper, a new method of quantitative analysis of college students' mental health risks is constructed by combining triangular fuzzy numbers and the entropy weight analytical method, and the degree of mental health of college students is judged by triangular fuzzy numbers. Meanwhile, entropy weight is used to rank the risk degrees of different universities. Compared with similar official data and empirical analysis, the fuzzy entropy weight method has good application value for the quantitative assessment of the mental health safety risk of college students.

\section{Data Availability}

The labeled datasets used to support the findings of this study are available from the corresponding author upon request.

\section{Conflicts of Interest}

The author declares no conflicts of interest.

\section{Acknowledgments}

This work was supported in part by the Jiangsu Province Education System Party Building Research Key Project "Research on the Construction of Quality Assurance System for the Development of University Student Party Members from the Perspective of Comprehensively Governing the
Party with Strict Discipline” (grant 2019JSJYDJ01018), Jiangsu University Philosophy and Social Science Research Project "Research on Ideological and Political Education Mechanism in Universities Based on Collaborative Perspective" (grant 2019SJB281), and Wuxi Vocational and Technical College Party Building Ideological and Political Annual Conference Topic (project no. 2019DJSZ12).

\section{References}

[1] S Zhang, T. Liu and F. Q. Xia, "A social network analysis approach to interpersonal relationship and mental health in college students," Chinese Mental Health Journal, vol. 34, no. 10 , pp. 855-859, 2020.

[2] C. L. Wu, S. T. Li, and L. Yan, "Research on the model of psychological crisis intervention for college students," Journal of Northeast Agricultural University, vol. 6, no. 6, pp. 28-30, 2008.

[3] M. Deng, "Application of fuzzy mathematics in mental health evaluation," Zhi Ye Quan, vol. 58, no. 6, pp. 91-92, 2007.

[4] W. G. Chen and X. F. Wang, "The design of a web-based psychology-health education expert system," Modern Educational Technology, vol. 13, no. 1, pp. 46-48, 2013.

[5] H. Feng, Y. Chen, and H. Liu, "Effects of positive psychological group training on psychological resilience and selfefficacy in special education teachers," Journal of Chinese Mental Health, vol. 34, no. 8, pp. 635-642, 2020.

[6] C. Y. Long and Q. Liu, "Construction of expert platform of student mental health education based on web," The Chinese Journal of ICT in Education, vol. 12, pp. 63-65, 2007.

[7] Ü. E. R. Sarp, Ö. C. E. B. E. Hilal, T. Gökhan Telatar et al., "Assessment of mental health of university students with GHQ12," Turkish Journal of Medical Sciences, vol. 38, no. 5, pp. 437-446, 2008.

[8] M. T. Kalkbrenner, "Recognizing and supporting students with mental health disorders: the REDFLAGS model," Journal of Education \& Training, vol. 3, no. 1, pp. 45-52, 2015.

[9] L. M. Farrer, A. Gulliver, N. Katruss et al., "A novel multicomponent online intervention to improve the mental health of university students: randomized controlled trial of the Uni Virtual Clinic," Internet Interventions, vol. 18, pp. 100-106, 2019.

[10] M. T. Kalkbrenner, E. M. Brown, and K. L. Carlisle, "Utility of the REDFLAGS model for supporting community college students' mental health: implications for counselors," Journal of Counseling \& Development, vol. 97, no. 4, pp. 417-426, 2019.

[11] A. Carlisle and M. L. Maryam, "Violence exposure and mental health of college students in the United States," Behavioral Sciences, vol. 8, no. 6, p. 53, 2018.

[12] F. A. Pompeo, "Mental health stigma among college students: misperceptions of perceived and personal stigmas," Journal of American College Health, no. 8, pp. 1-10, 2020.

[13] N. Abdurahman, "Reducing mental health stigma among multicultural high school students: educational and face-toface contact interventions," Dissertations \& Theses-Gradworks, pp. 245-255, 2015. 
[14] J. R. Moore, D. E. Pollio, B. A. Hong et al., "Pilot design and implementation of an innovative mental health and wellness clinic at a historically black college/university," Community Mental Health Journal, vol. 54, 2018.

[15] D. F. S. Craft, C. Travis, D. L. Martha et al., "Perceived and personal mental health stigma in latino and african American college students," Frontiers in Public Health, vol. 6, pp. 49-55, 2018.

[16] D. R. Mushonga, "The Glass Is Half Full: The Need to Promote Positive Mental Health in Black College Students," Journal of College Student Psychotherapy, no. 4, pp. 1-14, 2019.

[17] C. Y. Chan and K. L. Cheung, "Exploring the gender difference in relationships between narcissism, competitiveness, and mental health problems among college students," Journal of American College Health, no. 1, pp. 47-56, 2020.

[18] S. T. Chen, "Application of fuzzy comprehensive evaluation to psychological health assessment for university students," Journal of Jilin Engineering Normal University, vol. 34, no. 10, pp. 72-75, 2018.

[19] X. Y. Fang, X. J. Yuan, W. Hu et al., "The development of college students mental health screening scale," Studies of Psychology and Behavior, vol. 16, no. 1, pp. 111-118, 2018.

[20] D. Z. Li, Q. Li, and Y. M. Liu, "Fuzzy mathematics in evaluating college students' psychological status," College Mathematica, vol. 24, no. 4, pp. 23-26, 2008.

[21] X. Wang, "Research on mental health education for college students," International Journal of Social Ence and Education Research, vol. 3, no. 3, pp. 153-157, 2020.

[22] R. R. Fried, S. Karmali, and J. D. Irwin, "Minding many minds: an assessment of mental health and resilience among undergraduate and graduate students; a mixed methods exploratory study," Journal of American College Health, no. 11, pp. 1-13, 2020.

[23] L. He and S. Miao, "The design of the web-based multiple interactive psychological counseling platform," Modern Educational Technology, vol. 15, no. 6, pp. 43-46, 2005. 